\title{
Ngā Tikanga Whakamāori Kōrero
}

Nō te whakamanatanga o te Ture Reo Māori i te tau 1987 ka ara ake i tana moe te mahi nei o te whakamāori kōrero. Nā te ākinga e taua ture i puāwai ai te ahumahi whakamāori i ngā tau tata o muri mai, ā, mohoa nei e kaha tonu ana i te rāngai pāpāho, i te rāngai ture me te rāngai mātauranga. Hei tā Te Rōpū Kaiwhakamāori ā-waha, ā-tuhi o Aotearoa ${ }^{1}$ e tika ai tētehi whakamāoritanga me mau tonu te aronga ake o ngā kōrero ake, ā, kia kaua rawa atu te whakaaro e whakariroia, e tāpirihia, e tangohia rānei e te kaiwhakamāori. ${ }^{2}$ Ahakoa he aha te reo, ahakoa te momo reo, kei te kaiwhakamāori te haepapa ki te whakatutuki i tēnā whāinga, engari te kaiwhakamāori tuhinga pakimaero, ko te wātea ōna kei te āhua o tāna whakamāori i te tuhinga, inā hoki, kotahi te kōrero, manomano whakamāoritanga.

E rua ngā tino tikanga hei whāinga mā te kaiwhakamāori; ko tētehi ko te whakaahurea-tauiwi ${ }^{3}$ i te kōrero, arā, ko te mau tonu ki te ahurea me ngā ariā o te tuhinga ake, ahakoa pēwhea nei te rerekē o taua ahurea me aua ariā i ō te reo tuarua. Tēnā ko tēnei, ko te whakaahurea-māori ${ }^{4}$ i te kōrero, arā, ko te whakahāngai mai i te ahurea me ngā ariā o te tuhinga ake ki ō te reo tuarua (Yang, 2010). Ehara noa iho i te whakamāori ākupu, engari he whakamāori i te ahurea kia tau ai te noho o te kōrero i roto i te reo tuarua. Kāti, kia tirohia ètehi tauira o mua

\footnotetext{
${ }^{1}$ New Zealand Society of Translators and Interpreters.

2 NZSTI Code of Ethics and Code of Conduct (2013).

${ }^{3}$ Foreignization.

${ }^{4}$ Domestication
}

Hēmi Kelly holds a PhD in Māori and Indigenous Development from Auckland University of Technology. 
me ètehi o nā tata nei i whakamāorihia mai i te reo Pākehā e whai ana i ēnei tikanga whakamāori e rua. Mā te pēnā e mōhiotia ai me pēwhea te kaiwhakamāori tuhinga pakimaero i ēnei rā.

Ko te Paipera Tapu te pukapuka kua kaha rawa te whakamāorihia, huri i te ao. Kua eke tonu ki te 3000 ngā reo o tēnei pukapuka (Wycliffe Global Alliance, 2017). Nō te tau 1827 i tāia tuatahitia ai ètehi wāhanga o te Paipera Tapu i Poihākena. He mea whakamāori aua wāhanga nā ngā mihingare, nā Henry "Karuwhā" rāua ko tana teina, ko William "Parata" Williams (New Zealand Bible Society, 2017). Kei whea rā he tauira i tua atu i tā rāua i waiho mai ai hei whakaatu i te tikanga o te whakaahurea-tauiwi i te kōrero? Ahakoa he rerekē noa atu tā te Karaitiana titiro ki te ao, i tā te Māori, kāore ngā koroua rā i paku whai ki te whakaahurea-māori i ngā kōrero. Heoi anō, he whāinga anō tā ngā mihingare i pēnā ai rātou. Nā te pēnā i ako ai ngā iwi Māori i ngā tikanga me ngā ariā à-ahurea o te whakapono Karaitiana.

Tekau mā toru tau i muri mai ka riro mā Karuwhā rāua ko tana tamaiti, ko Edward Marsh "Eruera Maihi" Williams te Tiriti o Waitangi e whakamāori i te pō kotahi. Ki a au nei, i whakawhenumihia e rāua ngā tikanga whakamāori e rua. I te kōwae tuatahi i whakaahurea-tauiwitia te tuhinga $i$ te whakamahinga o te kupu "kāwanatanga" me ōna tikanga, ā, i te kōwae tuarua i whakaahurea-māoritia te tuhinga $i$ te whakamahinga o te kupu "tino rangatiratanga" me ōna tikanga. E rua, e rua i te kōrero i te mana whenua, engari he kupu kē, he ahurea kē, he aronga kē. Ehara te Tiriti i te tauira pai o te whakamāoritanga nā te kaha o te rerekē o te aronga o tētehi tuhinga i tētehi, engari he whakaatu noa atu tēnei $\mathrm{i}$ te pōraruraru ka pā pea ki te kaipānui i te whenumitanga o ènei tikanga e rua.

\section{Ko te tuatahi}


Ko nga Rangatira o te wakaminenga me nga Rangatira katoa hoki ki hai i uru ki taua wakaminenga ka tuku rawa atu ki te Kuini o Ingarani ake tonu atu - te Kawanatanga katoa o o ratou wenua.

\section{Ko te tuarua}

Ko te Kuini o Ingarani ka wakarite ka wakaae ki nga Rangitira ki nga hapu - ki nga tangata katoa o Nu Tirani te tino rangatiratanga o o ratou wenua o ratou kainga me o ratou taonga katoa. (New Zealand History, 2017)

Ko te tohungatanga o te kaiwhakamāori, kei te mātau ōna ki ngā reo e rua (Kratz, 1986). Ko te whakamāoritanga tuatahitanga o tētehi pakimaero ko Te Haerenga o te Manene (The Pilgrams Progress) i oti i a Eruera Maihi i te tau 1854. Kowata mai ana te kitea o te mātau o te tangata nei ki ngā reo e rua i tāna nei mahi. I noho reo rua ai ngā tuhinga o aua tau rā, tae atu ki te pito o te rautau 1800, nā ngā koroheke mihingare Pākehā nei, me te aha, i mihia anō rātou me tā rātou i mahi ai e ngā Māori i ngā tau o muri mai. He mihi tēnei nā Wiremu Rangihuna ki te tamaiti a Parata, ki a William Leonard "Mita Rēnata" Williams:

Tētahi o nga tino mahi nunui i mahia e Mita Rēnata ko te whakamāoritanga o ngā Karaipiture; e rāua ko tōna matua ko Wiremu Wiremu me Te Mānihera hoki. E kōrero nei ngā Māori katoa o Niu Tìrani i ngā Kawenata e rua, Mōmona, Pikopo, Weteriana, Perehipitini, Hauhau me èrā atu karangatanga huhua i waenganui i a tāua i te Maori he torutoru pea kei te mātau ko Pihopa Wiremu te tangata nāna i whakamāori, i whakatikatika haere hoki te whakamāoritanga. Kei whea te tangata reo reka i te Pīhopa mō te kōrero i te reo Māori kei runga noa ake tōna pai i te iwi nō rātou te reo. (Te Kopara, 1916) 
Ko tētehi Pākehā i hau ōna rongo i āna mahi whakamāori ko George Clarke "Hōri Karaka". Nō te tau 1824 ka ū mai ia ki Kerikeri, ā, ko ia te kaiwhakamāori i te whakawākanga o Maketū Wharetōtara nō Ngāpuhi i te tau 1842. Ko Maketū te tangata tuatahi i Aotearoa i tāronahia i raro i te ture mōna i kōhuru i ētehi tāngata tokorima (Grover, 1990). I ngā tau o muri mai i riro mā Hōri Karaka te niupepa o Te Karere Māori (18491854) e ētita, e whakamāori anō hoki. He tīpakohanga tēnei nō taua niupepa:

\section{LOST}

BEWTEEN the "Royal George" and Mr. Sharp's house, Mount Hobson, on Wednesday, 14th instant, a PARCEL, containing boys' clothes.

2 Black Cloth Coats,

2 do. do. Vests,

2 Pairs Black Cloth Trowsers,

2 do. Tweed Trowsers,

2 do. Braces.

Any person bringing the same to the office of this paper will be rewarded.

September 22, 1855

\section{MAHUE NOA}

KI TE TI, ki te ara tika atu rā nei ki Remuera, i te Wenetei, $i$ te 14 o te Marama, he TAKAI kākahu tamaiti, ko ngā taonga ènei o roto:-

2 Koti Mangu

2 Kōuma Mangu

2 Tarau Mangu

2 Tarau kara kē

2 Tākawe

Ki te kitea ēnei mea e te tangata, à, ka mauria mai ki te whare o tēnei nūpepa, ka riro atu he utu.

Hepetema 22, 1855. (The Maori Messenger - Ko te Karere Maori, 1855)

I konei, kua whakaahurea-māorihia te tuhinga i te āhua o tā Karaka whakamāori i ngā wāhi; kei te takiwā o Te Tī (Newmarket) te hōtēra o te "Royal George", ā, kei Remuera (Mt Hobson) te whare o Mr. Sharp. Nā te pēnei i mōhio ai ngā kaipānui Māori ko whea a whea - he reo kē, he ahurea kē, he aronga kē. Nō reira, kia hoki anō ki tāku i kī rā, ko te tohungatanga o te kaiwhakamāori kei te mātau ōna ki ngā reo e rua, ka tahi; ka rua, kei te mātau anō hoki ōna ki ngā ahurea o ngā iwi e rua - e rua, e rua he whaitake.

Nō te tau 1865 ka whakatūria te Kōti Whenua Māori. He nui ngā kaiwhakawā i aua tau i matatau ki te reo Māori, ko te 
nuinga hoki o ngā kaiwhakawā he mihingare (Boast, 2015). Mehemea kāore te kaiwhakawā i matatau ki te reo Māori i whakanōhia atu he kaiwhakamāori i roto i te kōti, ā, ko Charles Davis "Hāre Rēweti" tētehi o ngā kaiwhakamāori o te Kāwanatanga i te rohe o Tāmaki-makau-rau (Ward, 1990). He tangata i mōhiotia nuitia, i paingia nuitia hoki e ngā iwi Māori o taua rohe mōna i āwhina i ngā take hoko whenua, otirā, i ngā take Māori katoa (Ward, 1990).

Nō te tau 1881 i oti i a ia ètehi o ngā tuhituhi a Wīremu Hakipea te whakamāori, he mea whakaputa i te niupepa o $\mathrm{Te}$ Korimako i te tau 1884. I taua whakaputanga i àta tuhi ia e tika ana kia tukuna ēnei whakamāoritanga nāna hei whakaaetanga, hei whakahētanga, hei whakatikatikahanga rānei mā ngā kaipānui Māori. Ā, kāti, me tāpiri anō tēnā ki ngā pūkenga o te kaiwhakamāori kua kōrerotia, arā, te āheinga o te tangata ki te tuku i tāna i mahi ai hei kanohi hōmiromirotanga mā ngā karu o tētehi atu. Koinei tāna whakamāoritanga o tētehi wāhanga nō Othello (3.3.155-161):

GOOD NAME.

Who steals my purse, steals trash;

Tis something, nothing; 'twas mine,

'Tis his, and has been slave to thousands;

But he that filches from me my good name

Robs me of that which not enriches him, And makes me poor indeed.

\section{INGOA PAI.}

Ko ia e keiā ana i tōku rawa, $\mathrm{E}$ tango ana $\mathrm{i}$ te korekore noa;

He rawa nei anō, he kore hoki rā.

I a au anō rā hoki, kua riro atu māna;

$\bar{A}$, kua rōrātia ki te mano

Engari rā, ko ia kei te whēnako

I tōku ingoa pai, e muru ana I te rawa kāore nei e riro atu Hei nui mōna; ko ahau ia $\overline{\mathrm{E}}$, ka rawa kore noa. (Te Korimako, 1884)

Mō te whakamāoritanga kāore pea he tauira kei runga atu te mōhiotia i te waiata ā-motu o Aotearoa. Nō te tau $1878 \mathrm{ka}$ tono a Kāwana Kerei kia whakamāorihia te waiata o God Defend 
New Zealand nā Thomas Bracken (Te Parakena) i tito, ā, i riro nā Thomas Smith i mahi mai, he kaiwhakawā anō ia i roto i te Kōti Whenua Māori i te rohe o Tāmaki-makau-rau (Manatū Taonga, 2015). E rima katoa ngā whiti o te waiata nei; anei ngā whiti e rua o te tīmatanga:

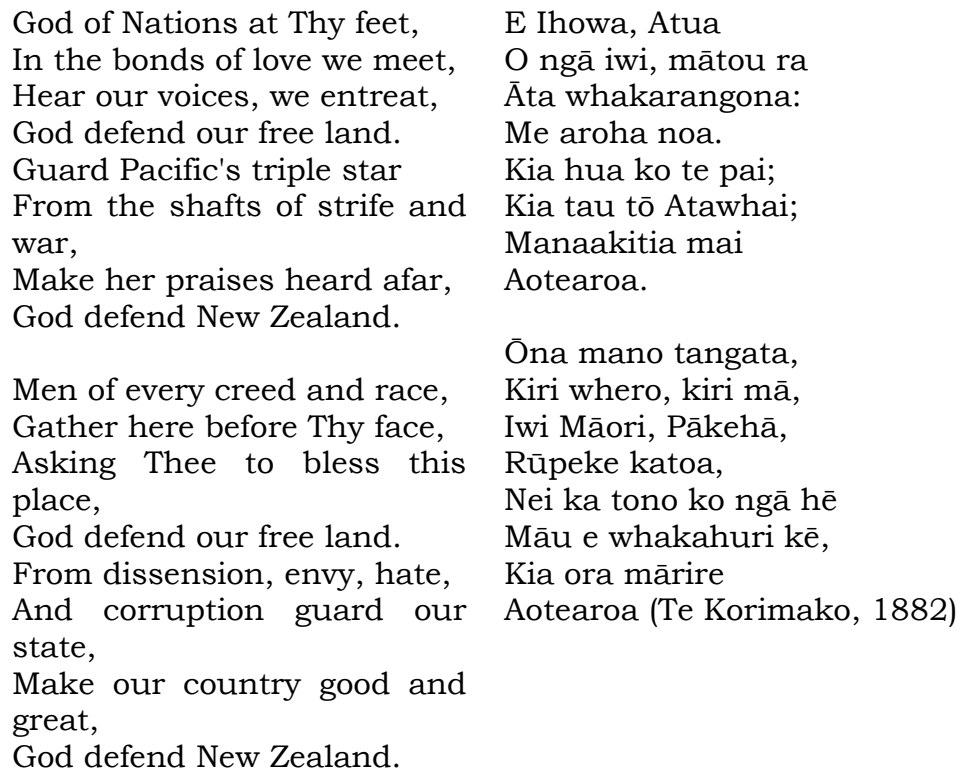

I konei i mate a Smith ki te āta whakarite i tāna whakamāoritanga ki te rangi o te waiata o ngā kupu Pākehā, engari nā taua here me tana whai kia noho ngā kupu i roto i te rangi Pākehā i puta pēnei mai ai tana whakamāoritanga. Me mihi te auaha, ahakoa he kupu kē, he kōrero kē, ko te wairua i mau tonu me te huarite anō o ngā kupu. Hei tā Yang (2010), mai iho he rite tonu te taupatupatuhia ko ēwhea o ēnei tikanga whakamāori te mea tika hei whāinga, ko te whakamāori noa iho i ngā kupu ${ }^{5}$, ko te whakamāori rānei i te wairua o te kōrero. ${ }^{6}$ I

\footnotetext{
${ }^{5}$ Literal (word-for-word) translation.

${ }^{6}$ Free (sense-for-sense) translation.
} 
te nuinga o te wā, e paingia ana te tikanga tuarua nei me he toikupu, pēnei i te waiata nei.

Nō te huringa mai o te rautau 1900 kātahi ka tokomaha ake ngā kaiwhakamāori Māori i ngā kaiwhakamāori Pākehā. Ko te reanga tēnei i a Tā Apirana Ngata mā, i a Kīngi Tahiwi mā, i tae ki ngā kura ki te rapu i te hōhonutanga o te mātauranga o te Pākehā, ā, ka hono i roto i a rātou te ao Māori me te ao Pākehā. Ko te tokorua o taua reanga hei kōrerotanga māku ko Rēweti Kōhere rāua ko Pei Te Hurinui Jones. Hei tā Kōhere, he mahi ngahau nāna "te tuhituhi i ngā kōrero a ngā ihumanea o te Pākehā" (Te Toa Takitini, 1931) I oti i a ia te whakamāori ngā tuhinga huhua a ngā tino tohunga tuhituhi o te ao Pakeha. Koinei tāna whakamāoritanga o tētehi kōrero nā Alexander Pope:

A little learning is a Kia tūpato ki te mātauranga dangerous thing, pakupaku, Kia hōhonu te inu, Drink deep or taste not the ki te kore kaua e pā atu ki te Pierian spring." puna o te mātauranga. (Te Toa Takitini, 1924)

Anei anō tētehi nā Robert Burns:

The rank is but the guinea's stamp, The man's the gowd for a' that.
Ko te ingoa o te tangata e rite ana ki te tuhituhi o te moni, Ko te tangata anō ia te koura aha he aha te aha. (Te Toa Takitini, 1924)

Kāore kau he whakamārama mō te Pierian Spring mō te guinea stamp ranei i ngā tāhuhu kōrero a te Māori, nō ahurea kē ēnā kōrero. He nuku atu i te whakamāori kupu tā Kōhere mahi i ēnei tauira e rua, he whakamāori tāna i te wairua o te kōrero, i te ahurea me ngā ariā o roto i te kōrero ake kia māramatia ai i te reo Māori. Engari, kāore te katoa o tōna reanga i whai i tērā tikanga. I oti i a Pei Te Hurinui te whakamāoritanga o Te Tangata Whairawa o Wēniti (The 
Merchant of Venice) i te tau 1927. He wā ōna i rite ā Jones mahi ki ā Kōhere i runga ake nei, engari he wā anō i ù kē ia ki te whakaahurea-tauiwi i te kōrero kia kore ai e ngaro te ahurea ake o te tuhinga ake, hei tauira:

I tell you this...When, E kōrero atu ana ahau ki a koe started from the Goal, i tēnei...

I te tīmatatanga mai $\mathrm{i}$ te $\mathrm{Pou}$ tapu,

Over the flaming shoulders Whiti rawa mā runga i ngā of the Foal pakihiwi wera ō te Kūao

Of Heav'n Parwin and Ō te rangi; Nā Pāwinia rāua ko Mushtari they flung, Māhutari nā rāua i whiu, In my predestin'd plot of Te Puehu me Te Wairua ki roto Dust and Soul i tōku Urupā kua tohuria mai i te Orokotimatanga (Fitzgerald \& Jones, 1975, p. 81).

He tīpakohanga tēnei nō tāna whakamāori i Ngā Rūpai'aha a Ōmā Kai'ama (Rubáiyát of Omar Khayyám). Nō te reo tawhito o Pāhia ēnei ingoa o Pāwinia rāua ko Māhutari. Ki te iwi Muhirama ko Pāwinia te ingoa o Pareārau, ā, ko Māhutari te ingoa o Matariki. Mehemea a Jones i whakamahi i ngā ingoa Māori ake o aua whetū kua uru mai he wairua kē, he whakamārama kē ki te tuhinga, kua kore te kōrero e noho noa iho i roto $i$ te ahurea tawhito o te Muhirama, engari kua tōia mai ngā kōrero rā ki roto ki te ao Māori. Mā te mau tonu ki ngā ingoa ake o taua ahurea me te kore i whakahāngai mai i ètehi o ngā kōrero ki ā te Māori e kitea ai tētehi ao kē atu me tōna ahurea mā roto tonu mai i tō tātou reo. Ehara tēnā i te mahi takoto noa.

Nō muri mai i te momo i ēnei koroheke i whakamārari haere ngā mahi whakamāori kōrero, tae noa atu ki te takiwā o te whakamanatanga o te Ture Reo Māori; i reira ka ara mai anō. I te tau 1986 i whakamāorihia e Jean Wikiriwhi te pukapuka pakimaera tuatahi nā te Māori i tuhi ki te reo Pākehā, arā ko 
Pounamu, Pounamu nā Witi Ihimaera. I te tau 1995 i whakamāorihia tētehi anō pukapuka nāna, arā ko Te Kaieke Tohorā, e Tā Tīmoti Kāretu. Nā te mea kei te ahuea Māori kē ēnei pukapuka e rua, kāore ngā kaiwhakamāori i mate ki te whiriwhiri i ngā tikanga e rua.

I te tau 2015, nā Te Haumihiata Mason i whakamāori a Rōmea rāua ko Hurieta. Koinei te tuarua o ngā tuhinga a Hakipea kia whakamāorihia mai i te tīmatanga ki te mutunga. I āta whai a Mason ki te whakaahurea-māori i te tuhinga ake, ā, nāna i whakahāngai mai te ahurea me ngā ariā o taua ao Pākehā o nehe ki ō te Māori o mua. Hei tauira, i te reo Pākehā ka puta te kōrero a Monataku (Montagues) ki a Kapureti (Capulets) i te tīmatanga, e whawhai ana rāua, "Do you bite your thumb at me?" Ko te ritenga o tēnei mahi o mua i ènei rā ko te whakatū pea i te māpere ki tētehi atu. Ko tā Mason whakamāori mai i taua kōrero, otirā i taua mahi ko tēnei nā, " $E$ whakapohane mai ana koe?” He whakaahurea-māori tāna i tēnei tuhinga rongonui o te ao Pākehā. Kia kī ake au, nāna, nā tana pērā i tino mārama ai au ki te kōrero mō Rōmeo rāua ko Hurieta.

I ngā tau tata nei kua kitea ètehi tuatahitanga hei whakahīhītanga mā tātou. Kua whakamāorihia ètehi o ngā pakiwaituhi rongonui pēnei i a Tarau Porowhā (SpongeBob Square Pants) me Tōra Mãtātoa (Dora the Explorer) e Pānia Papa rāua ko Heketū Blake me ngā kaiāwhina i a rāua, ā, nō tēnei tau tonu nei i whakakitea ai te kiriata tuatahi nā Disney ki te reo Māori, arā ko Moana. E hīkaka ana te ngākau, nā te mea e ahu atu ana tātou ki tētehi wāhi kāore anō tātou kia pā atu.

Hāunga tēnā, e makaia ana te kōrero e ètehi e mea ana, hei aha noa iho i whakapau kaha ai ki te whakamāori i ngā mahi a ètehi atu, me whakapau kē i ō tātou kaha ki te whakaputa i à tātou mahi ake ki te reo Māori tonu. Me pēnā ka tika, engari me kaha ake anō hoki ngā mahi whakamāori kia nui noa atu ngā matatiki hei toro atu mā te hunga kōrero Māori. Mā te whakamāori e ora tonu mai ai ngā mahi a ètehi atu, mā te whakamāori e hōpara ai tātou i te ao whānui mā roto tonu mai 
i tō tātou reo. Waihoki, he tohu o te reo e ora ana; whakaarotia ake te nui o ngā whakareopākehātanga i roto i ngā tau.

Tērā te wā i kaha tēnei mahi i Aotearoa, nā ngā mihingare Pākehā i tīmata ai, nā rātou anō hoki i kawe tae noa atu ki te huringa mai o te rautau 1900. Kātahi ka hāpaitia ake e ngā koroheke Māori i puta i ngā kura, à, ināianei kua riro mā tēnei reanga e hāpai ake. Nō reira, me tuku pātai, he aha tā tātou hei waihotanga iho ki ngā reanga o muri mai? Ko te uaua kei mua i te kaiwhakamāori tuhinga pakimaero ko te here o te wā; e pokea kētia ana te nuinga o ngā kaiwhakamāori e ngā mahi i roto $i$ te rāngai ture, $i$ te rāngai pāpāho me te rāngai mātauranga, i te tukunga iho, kāore nei e aronuitia ana ngā mahi ngahau nei i kōrerotia rā e Kōhere.

I tua atu i tenā, ko te mahi nui a te kaiwhakamāori tuhinga pakimaero ko te whiriwhiri me pēwhea rā te āhua o te whakamāori; me pēwhea te kaha o te whakaahurea-tauiwi, me pēwhea rānei te kaha o te whakaahurea-māori i ngā kōrero. He painga ō ngā tikanga e rua: mā te whakaahurea-tauiwi e ako ai tātou i ngā tikanga me ngā ariā ā-ahurea o iwi kē me te āhua o tā iwi kē titiro ki te ao mā roto tonu mai i te reo Māori; tēnā, mā te whakaahurea-māori e kitea ai tētehi kōrero nō waho mā roto i tā tātou titiro ki te ao i te mea kua oti ngā tikanga me ngā ariā à-ahurea te whakahāngai me te whakarite mai, mā reira pea e tau ai te māramatanga ki runga i ētehi kōrero tino tauhōu rawa atu ki tā te Māori titiro.

He whakamārama hei ārahi i te kaiwhakamāori kei roto i ngā tauira o mua kua tirohia i tēnei tuhinga, engari he korakora noa iho ēnei nō ngā kōrero katoa kua oti te whakamāori. Mā te titiro ki ēnei tauira e kitea ai te pēwheatanga; he aha ngā tauira hei whāinga, he aha hoki ngā tauira kia kaua e whāia atu. Ki a au nei, mā te horopaki e tohu mai ko tēwhea o ngā tikanga hei whāinga. Ahakoa pēwhea kāore e taea te kore nei i whakaahurea-māori i te kōrero, inā hoki, he whakapapa, he kōrero, he whakaaro, he ahurea Māori kei roto i ngā kupu Māori. Engari, mā te kaiwhakamāori e whakatau ka pēwhea te kaha o tāna tō mai i te kōrero ki roto ki te ahurea Māori. 


\section{Ngā tohutoro}

Boast, R. (2015). Te tango whenua-Māori land alienation - Establishing the Native Land Court. Te Ara - the Encyclopedia of New Zealand. Retrieved from http://www.TeAra.govt.nz/en/te-tango-whenuamaori-land-alienation/page-5 
Fitzgerald, E., \& Jones, P. (1975). Ngā Rūpai'aha a Ōmā Kaiama.

Grover, R. (1990). Clarke, George. Te Ara - the Encyclopedia of New Zealand. Retrieved from https://teara.govt.nz/en/biographies/1c18/clarke-george

Kratz, D. (1986). An Interview with Norman Shapiro. Translation Review, Vol. 19, Iss. 1.

Manatū Taonga - Ministry of Culture \& Heritage. (2015). History of God Defend New Zealand. Retrieved from https:/ /www.mch.govt.nz/nzidentity-heritage/national-anthems/history-god-defend-newzealand

New Zealand Bible Society. (2017). The Bible in Māori. Retrieved from https:/ / biblesociety.org.nz/discover-the-bible/the-bible-in-maori/

New Zealand History. (2017). Read the Treaty. Retrieved from https://nzhistory.govt.nz/politics/treaty/read-the-treaty/maoritext

Te Korimako. (1884, April 15). Shakespeare. Retrieved from http: / /www.nzdl.org/gsdlmod?e=d-00000-00---off-0niupepa--000----0-10-0---0---Odirect-10---4-------0-11pc--11-mi-50---20about-othello--00-0-1-00-0--4----0-0-11-10-0utfZz-8$00 \& \mathrm{cl}=$ search\&d=25_0_26.8\&gg=full

Te Kopara. (1916, September 15). Whakahemonga o nga kaumatua tawhito. Retrieved from http: / /www.nzdl.org/gsdlmod?gg=prev\&e=d-00000-00---offOniupepa--00-0----0-10-0---0---Odirect-10---4-------0-11pc--11-mi50---20-about-\%22reo+reka+i+te+pihopa\%22--00-0-1-00-0--4---$0-0-11-10-0 u t f Z z-8-00 \& a=d \& c l=\& d=40 \_35.5$

Te Korimako. (1882, May 16). Retrieved from http: / / www.nzdl.org/gsdlmod?gg=full\&e=d-00000-00---offOniupepa--00-0----0-10-0---0---0direct-10---4-------0-11pc--11-mi50---20-about-parakena--00-0-1-00-0--4----0-0-11-10-0utfZz-8$00 \& \mathrm{a}=\mathrm{d} \& \mathrm{cl}=$ search $\& \mathrm{~d}=25 \_0 \_3.2$

Te Toa Takitini. (1931, December 1). Nga kupu a Anaru Kaneki te mea nui ake $i$ ana miriona. Retrieved from http: / / www.nzdl.org/gsdlmod?gg=full\&e=d-00000-00---offOniupepa--00-0----0-10-0---0---0direct-10---4-------0-11pc--11-mi50---20-about-ihumanea--00-0-1-00-0--4----0-0-11-10-0utfZz-8$00 \& a=d \& c l=$ search $\& d=41 \_1 \_6.7$ 
Te Toa Takitini. (1924, October 1). He Kupu Tohunga. Retrieved from http: / / www.nzdl.org/gsdlmod?gg=full\&e=d-00000-00---off-

Oniupepa--00-0----0-10-0---0---Odirect-10---4-------0-11pc--11-mi50---20-about-\%22matauranga+pakupaku\%22--00-0-1-00-0--4---0-0-11-10-0utfZz-8-00\&a=d\&cl=search\&d=41_39.2

The Maori Messenger, Ko te Karere Maori. (1855, December 15). Lost Mahue noa. Retrieved from http://www.nzdl.org/gsdlmod?e=d00000-00---off-Oniupepa--00-0----0-10-0---0---Odirect-10---4------0-11pc--11-mi-50---20-about-\%22kouma+ma\%22--00-0-1-00-0-4----0-0-11-10-0utfZz-8-00\&cl=CL1.3\&d=02_5_130.1\&gg=full

Venuti, L. (1995). The Translator's Invisibility - A History of Translation. Routledge, London.

Ward, A. (1990). Davis, Charles Oliver Bond. Te Ara - the Encyclopedia of New Zealand. Retrieved from https://teara.govt.nz/en/biographies/1d3/davis-charles-oliverbond

Wycliffe Global Alliance. (2017). Scripture \& Language Statistics 2017. Retrieved from http://www.wycliffe.net/statistics

Yang, W. (2010). Brief Study on Domestication and Foreignization in Translation. Journal of Language Teaching and Research, Vol. 1, No. 1, pp. 1-7. 